ANNALES

POLONICI MATHEMATICI

$83.1(2004)$

\title{
Formal relations between quasianalytic functions of some fixed class
}

\author{
by F. Broglia (Pisa), A. Elkhadiri (Kenitra) and F. Pieroni (Pisa)
}

\begin{abstract}
In [Ga] Gabrielov has given conditions under which the completion of the kernel of a morphism $\varphi: A \rightarrow B$ between analytic rings coincides with the kernel of the induced morphism $\widehat{\varphi}: \widehat{A} \rightarrow \widehat{B}$ between the completions. If $B$ is a domain, a sufficient condition is that $\operatorname{rk} \varphi=\operatorname{dim}(\widehat{A} / \operatorname{ker} \widehat{\varphi})$, where $\operatorname{rk} \varphi$ is the rank of the jacobian matrix of $\varphi$ considered as a matrix over the quotient field of $B$. We prove that the above property holds in a fixed quasianalytic Denjoy-Carleman class if and only if the class coincides with the ring of germs of analytic functions.
\end{abstract}

1. Introduction. The Denjoy-Carleman classes are classes of $C^{\infty}$ functions defined by imposing some bounds on the growth of their derivatives (see Section 2 for the definition); these classes contain the analytic functions. The Denjoy-Carleman Theorem characterizes the classes which are quasianalytic, i.e. which do not contain flat functions.

These classes, in one variable, were introduced by E. Borel a century ago; soon they became an important tool in harmonic analysis and when studying linear partial differential equations. They are less understood in dimension $\geq 2$.

Recently Rolin, Speissegger and Wilkie [RSW] found an o-minimal expansion of the real field described by these functions when restricted to the closed cube and extended by zero outside.

The rings of germs of these functions share some good properties with the analytic germ ring, for instance they are henselian and closed under differentiations. It is still unknown (and unlikely) whether they are noetherian, and this fact makes it impossible to use standard algebraic techniques. Nevertheless several properties can be obtained; for instance Bierstone and

2000 Mathematics Subject Classification: Primary 32B20.

Key words and phrases: Denjoy-Carleman class, Gabrielov property, quasianalytic.

Recherche de A. Elkhadiri menée dans le cadre du projet PARS MI 33.

F. Broglia and F. Pieroni are members of GNSAGA of CNR, partially supported by MURST and by the European Research Training Network RAAG 2002-2006 (HPRN-CT00271). 
Milman $[\mathrm{BM}]$ proved a resolution of singularities, topological noetherianity, Łojasiewicz inequality and a curve selection lemma. Elkhadiri [El] proved a theorem of the complement for quasi subanalytic sets.

On the other hand, Childress [Ch] showed that the Weierstrass Division Theorem does not hold unless the class is analytic.

In this short note we prove a similar implication for a property that was proved by Gabrielov for convergent power series.

Consider a map $\varphi:\left(\mathbb{R}^{n}, 0\right) \rightarrow\left(\mathbb{R}^{k}, 0\right)$ vanishing at zero, where $\varphi$ is a $k$-tuple $\left(\varphi_{1}, \ldots, \varphi_{k}\right)$ and $\varphi_{1}, \ldots, \varphi_{k}$ are germs of functions in a fixed quasianalytic Denjoy-Carleman class. It defines uniquely a map $\varphi^{*}$ between the rings of germs by composition, hence it induces a morphism $\widehat{\varphi}^{*}$ between their completions. Gabrielov studied this situation for analytic rings and gave conditions for the completion of $\operatorname{ker} \varphi^{*}$ to be the kernel of $\widehat{\varphi}^{*}$. We prove that the same statement does not hold for the ring of germs of a fixed quasianalytic Denjoy-Carleman class unless the class is analytic.

The proof will be carried out in several steps. First, we prove as in $[\mathrm{EH}]$ that the result of Gabrielov implies a result on "composition" (Lemma 3.3); then we use this, in particular a quasianalytic version of the Newton Theorem, to get the so called generic division theorem (Proposition 3.5; cf. $[\mathrm{Gl}]$ ), and we obtain the assertion as a consequence of the result of Childress.

2. Definitions and recalls. Let $\mathcal{E}(U)$ denote the ring of $C^{\infty}$ functions on an open set $U$ of $\mathbb{R}^{n}$. As usual, for each $n$-tuple $\alpha=\left(\alpha_{1}, \ldots, \alpha_{n}\right)$ of nonnegative integers, we set $|\alpha|=\alpha_{1}+\ldots+\alpha_{n}$ and $D^{\alpha}=\partial^{|\alpha|} / \partial_{x_{1}}^{\alpha_{1}} \ldots \partial_{x_{n}}^{\alpha_{n}}$.

Let $\varrho>0$ and let $M=\left\{M_{p}\right\}_{p=0}^{\infty}$ be an increasing sequence of positive real numbers. For each $f \in \mathcal{E}(U)$,

$$
\|f\|_{U, \varrho, M}=\sup _{x \in U, p \in \mathbb{N}} \sup _{|\alpha|=p} \frac{\left|D^{\alpha} f(x)\right|}{\varrho^{p} M_{p} p !}
$$

defines a norm on

$$
C_{M, \varrho}(U)=\left\{f \in \mathcal{E}(U) \mid\|f\|_{U, \varrho, M}<\infty\right\},
$$

which makes it a Banach space. We say that $f \in \mathcal{E}(U)$ is in the DenjoyCarleman class defined by $M$, briefly $f \in C_{M}(U)$, if $f \in C_{M, \varrho}(U)$ for some $\varrho>0$. Finally, we denote by $C_{M}\left(\mathbb{R}^{n}, 0\right)$ the class of germs of functions $f \in C_{M}(U)$ at the origin of $\mathbb{R}^{n}$.

In order to get classes of functions with some structural properties, we impose some conditions on the sequence $M=\left\{M_{p}\right\}_{p=0}^{\infty}$ (we follow, for instance, $[\mathrm{BM}]$ and $[\mathrm{Ch}])$ :

$$
M=\left\{M_{p}\right\}_{p=0}^{\infty} \text { is logarithmically convex and } M_{0}=1 ;
$$




$$
\begin{aligned}
& \sup _{p}\left(\frac{M_{p+1}}{M_{p}}\right)^{1 / p}<\infty \\
& \sum_{p} \frac{M_{p}}{(p+1) M_{p+1}}=\infty .
\end{aligned}
$$

The first hypothesis ensures that $C_{M}\left(\mathbb{R}^{n}, 0\right)$ is a local ring with maximal ideal $\mathfrak{m}=\left\{f \in C_{M}\left(\mathbb{R}^{n}, 0\right) \mid f(0)=0\right\}$ and that it is closed under composition. This means that if $g \in C_{M}\left(\mathbb{R}^{k}, 0\right)$ and $f=\left(f_{1}, \ldots, f_{k}\right) \in\left(C_{M}\left(\mathbb{R}^{n}, 0\right)\right)^{k}$ with $f(0)=0$, then $g \circ f \in C_{M}\left(\mathbb{R}^{n}, 0\right)$. Moreover, this hypothesis implies that $M_{p}^{1 / p}$ is not decreasing, and this simply means that $C_{M}\left(\mathbb{R}^{n}, 0\right)$ contains $\mathbb{R}\left\{x_{1}, \ldots, x_{n}\right\}$.

Adding the second condition we get that $C_{M}\left(\mathbb{R}^{n}, 0\right)$ is closed under division by coordinates, i.e. if $f\left(x_{1}, \ldots, x_{i-1}, a_{i}, x_{i+1}, \ldots, x_{n}\right)$ is identically zero then $f(x)=\left(x_{i}-a_{i}\right) g(x)$ with $g \in C_{M}\left(\mathbb{R}^{n}, 0\right)$, and under differentiation. Note that, in general, if $f=\psi g \in C_{M}\left(\mathbb{R}^{n}, 0\right)$, also with $\psi \in \mathbb{R}\left\{x_{1}, \ldots, x_{n}\right\}$, the regularity of $g$ is unknown.

The last property is equivalent to the so called quasianalyticity, i.e. it ensures that the map

$$
T: C_{M}\left(\mathbb{R}^{n}, 0\right) \rightarrow \mathbb{R}\left[\left[x_{1}, \ldots, x_{n}\right]\right]
$$

which associates to each function germ $f$ its Taylor series $T f$ at the origin is injective.

Moreover, under our assumption on $M$, the Implicit Function Theorem for $C_{M}\left(\mathbb{R}^{n}, 0\right)$ also holds (cf. [BM], [Ko]):

TheOREM 2.1. Let $f=\left(f_{1}, \ldots, f_{m}\right) \in\left(C_{M}\left(\mathbb{R}^{m+n}, 0\right)\right)^{m}$ with $f(0,0)=0$. Suppose that

$$
\operatorname{det}\left(\frac{\partial f_{i}}{\partial x_{j}}\right)_{i, j=1, \ldots, m}(0,0) \neq 0 .
$$

Then there is a (unique) $g=\left(g_{1}, \ldots, g_{n}\right) \in\left(C_{M}\left(\mathbb{R}^{m}, 0\right)\right)^{n}$ with $g(0)=0$ such that

$$
f(x, g(x))=0
$$

3. Gabrielov's property. Let $M=\left\{M_{p}\right\}_{p=0}^{\infty}$ be an increasing sequence of positive real numbers satisfying conditions $(1)-(3)$. Let $\varphi=\left(\varphi_{1}, \ldots, \varphi_{k}\right)$ with $\varphi_{i} \in C_{M}\left(\mathbb{R}^{n}, 0\right)$ and $\varphi(0)=0$, and let $x=\left(x_{1}, \ldots, x_{n}\right)$ and $y=$ $\left(y_{1}, \ldots, y_{k}\right)$ be the coordinates in $\mathbb{R}^{n}$ and $\mathbb{R}^{k}$ respectively. The generic rank of $\varphi, \operatorname{rk} \varphi$, is the rank of the matrix $\left(\partial \varphi_{i} / \partial x_{j}\right)$ considered as a matrix over the quotient field of $C_{M}\left(\mathbb{R}^{n}, 0\right)$ (recall that $C_{M}\left(\mathbb{R}^{n}, 0\right)$ is a domain).

We consider the morphism

$$
\varphi^{*}: C_{M}\left(\mathbb{R}^{k}, 0\right) \rightarrow C_{M}\left(\mathbb{R}^{n}, 0\right)
$$


defined by $\varphi^{*}\left(y_{i}\right)=\varphi_{i}$ and its extension to the completions

$$
\widehat{\varphi}^{*}: \mathbb{R}[[y]] \rightarrow \mathbb{R}[[x]] .
$$

Let $J \subset \mathbb{R}[[y]]$ be the ideal of all formal relations between the functions $\varphi_{i}$, i.e. the kernel of $\widehat{\varphi}^{*}$, and let $I \subset C_{M}\left(\mathbb{R}^{k}, 0\right)$ be the ideal of all quasianalytic relations, i.e. the kernel of $\varphi^{*}$. Following [Ga], we put

$$
r_{1}(\varphi)=\operatorname{rk} \varphi, \quad r_{2}(\varphi)=\operatorname{dim}(\mathbb{R}[[y]] / J)
$$

(dim is the Krull dimension). In general $I \mathbb{R}[[y]] \subset J$ and $r_{1}(\varphi) \leq r_{2}(\varphi)$, and already for an analytic morphism between analytic rings the inclusion and the inequality can be strict. Gabrielov has proved that if "sufficiently many" formal relations exist between analytic germs (i.e. $r_{1}(\varphi)=r_{2}(\varphi)$ ) then all of these are generated by analytic relations. This result justifies the following definition:

Definition 3.1. We say that the rings $C_{M}\left(\mathbb{R}^{m}, 0\right), m \in \mathbb{N}$, have the Gabrielov property if, given $\varphi^{*}: C_{M}\left(\mathbb{R}^{k}, 0\right) \rightarrow C_{M}\left(\mathbb{R}^{n}, 0\right)$ as above,

$$
r_{1}(\varphi)=r_{2}(\varphi) \Rightarrow J=I \mathbb{R}[[y]]
$$

that is, the completion of the kernel coincides with the kernel of the morphism induced between the completions.

The aim of this paper is to prove the following:

TheOREM 3.2. Let $M=\left\{M_{p}\right\}_{p=0}^{\infty}$ be an increasing sequence of positive real numbers satisfying conditions (1)-(3), such that the classes $C_{M}\left(\mathbb{R}^{m}, 0\right)$, $m \in \mathbb{N}$, have the Gabrielov property. Then $C_{M}\left(\mathbb{R}^{m}, 0\right)=\mathbb{R}\left\{x_{1}, \ldots, x_{m}\right\}$ for each $m \in \mathbb{N}$.

Keeping the same notations as above, we have the following:

Lemma 3.3. Let $C_{M}\left(\mathbb{R}^{m}, 0\right), m \in \mathbb{N}$, be quasianalytic classes with the Gabrielov property. Let $\varphi^{*}: C_{M}\left(\mathbb{R}^{k}, 0\right) \rightarrow C_{M}\left(\mathbb{R}^{n}, 0\right)$ be a morphism with $\operatorname{rk} \varphi=k$. Let $\widehat{f} \in \mathbb{R}\left[\left[y_{1}, \ldots, y_{k}\right]\right]$ be such that $\widehat{\varphi}^{*}(\widehat{f})=T g$ with $g \in C_{M}\left(\mathbb{R}^{n}, 0\right)$. Then there exists $f \in C_{M}\left(\mathbb{R}^{k}, 0\right)$ such that $T f=\widehat{f}$.

Proof. We can suppose $\widehat{f}(0)=0$. Since $\operatorname{rk} \varphi=k, \widehat{\varphi}^{*}$ and $\varphi^{*}$ are injective, hence $\operatorname{dim} \widehat{\varphi}^{*}(\mathbb{R}[[y]])=k$.

Introducing a new variable $t$, we can extend $\varphi^{*}$ to $\varphi_{t}^{*}$, and then $\widehat{\varphi}^{*}$ to $\widehat{\varphi}_{t}^{*}$, by putting $\varphi_{t}^{*}(t)=g$. We have $\widehat{\varphi}_{t}^{*}(\mathbb{R}[[y, t]])=\widehat{\varphi}^{*}(\mathbb{R}[[y]])$, hence $\operatorname{ht}\left(\operatorname{ker} \widehat{\varphi}_{t}^{*}\right)=1$. Since $\operatorname{ker} \widehat{\varphi}_{t}^{*}$ is a prime ideal, it is principal and $\operatorname{ker} \widehat{\varphi}_{t}^{*}=$ $(t-\widehat{f}) \mathbb{R}[[y, t]]$. Since $\operatorname{rk} \varphi=\operatorname{rk} \varphi_{t}$, where $\varphi_{t}=(\varphi, g)$, the Gabrielov property implies that $\operatorname{ker} \widehat{\varphi}_{t}^{*}=\left(\operatorname{ker} \varphi_{t}^{*}\right) \mathbb{R}[[y, t]]$.

Let $h_{1}, \ldots, h_{s}, \ldots \in C_{M}\left(\mathbb{R}^{k}, 0\right)$ be (maybe infinitely many) generators of $\operatorname{ker} \varphi_{t}^{*}$. Then there exist $m \in \mathbb{N}$ and $\widehat{\alpha}_{1}, \ldots, \widehat{\alpha}_{m} \in \mathbb{R}[[y, t]]$ such that

$$
t-\widehat{f}=\widehat{\alpha}_{1} T h_{1}+\ldots+\widehat{\alpha}_{m} T h_{m} .
$$


Since $T h_{1}, \ldots, T h_{m} \in \operatorname{ker} \widehat{\varphi}_{t}^{*}$, there exist $\widehat{q}_{1}, \ldots, \widehat{q}_{m} \in \mathbb{R}[[y, t]]$ such that $T h_{i}=\widehat{q}_{i}(t-\widehat{f}), i=1, \ldots, m$. Substituting, we obtain

$$
t-\widehat{f}=\left(\widehat{\alpha}_{1} \widehat{q}_{1}+\ldots+\widehat{\alpha}_{m} \widehat{q}_{m}\right)(t-\widehat{f}) .
$$

Thus, there exists at least one $i \in\{1, \ldots, m\}$ such that $\widehat{q}_{i}$ is a unit. Fix such an $i$ and set $h_{i}=h$ and $\widehat{q}_{i}=\widehat{q}$. We see that

$$
h(0,0)=0, \quad \frac{\partial h}{\partial t}(0,0) \neq 0 .
$$

By the Implicit Function Theorem, there exists $\psi \in C_{M}\left(\mathbb{R}^{k}, 0\right), \psi(0)=0$, such that

$$
h(y, t)=0 \Leftrightarrow t=\psi(y) .
$$

By the Implicit Function Theorem in the ring $\mathbb{R}[[y, t]]$, similarly we get $\widehat{\psi}(y) \in \mathbb{R}[[y]]$ such that $T h(y, \widehat{\psi}(y))=0$. Since such a $\widehat{\psi}(y)$ is unique, we have $\widehat{\psi}=\widehat{f}=T \psi$. This ends the proof with $f=\psi$.

If $\lambda=\left(\lambda_{1}, \ldots, \lambda_{p}\right) \in \mathbb{R}^{p}$, then $P\left(x_{n}, \lambda\right)=x_{n}^{p}+\sum_{i=1}^{p} \lambda_{i} x_{n}^{p-i}$ is called a generic polynomial in $x_{n}$ of degree $p$.

Let $\sigma=\left(\sigma_{1}, \ldots, \sigma_{p}\right): \mathbb{R}^{p} \rightarrow \mathbb{R}^{p}$ be the polynomial map defined by $\sigma_{i}=(-1)^{i} \widetilde{\sigma}_{i}$, where the $\widetilde{\sigma}_{i}$ 's are the elementary symmetric functions in the variables $\lambda_{1}, \ldots, \lambda_{p}$. Then we have the identity

$$
P\left(x_{n}, \sigma(\lambda)\right)=\left(x_{n}-\lambda_{1}\right) \ldots\left(x_{n}-\lambda_{p}\right) .
$$

Let $\varphi^{*}: C_{M}\left(\mathbb{R}^{n+p}, 0\right) \rightarrow C_{M}\left(\mathbb{R}^{n+p}, 0\right)$ be defined by $\varphi(x, \lambda)=(x, \sigma(\lambda))$. We can see that $\operatorname{rk} \varphi=n+p$. Lemma 3.3 implies a quasianalytic version of the Newton Theorem:

Corollary 3.4. Let $f(x, \lambda) \in C_{M}\left(\mathbb{R}^{n+p}, 0\right)$ be a function germ symmetric with respect to the variables $\lambda_{i}$. Then there exists $g \in C_{M}\left(\mathbb{R}^{n+p}, 0\right)$ such that $f(x, \lambda)=g(x, \sigma(\lambda))$.

LEMma 3.5. Let $P\left(x_{n}, \lambda\right)$ be a generic polynomial in $x_{n}$ of degree $p$. If $f \in C_{M}\left(\mathbb{R}^{n}, 0\right)$ then there exist unique function germs $q \in C_{M}\left(\mathbb{R}^{n+p}, 0\right)$ and $r_{j} \in C_{M}\left(\mathbb{R}^{n-1+p}, 0\right), 1 \leq j \leq p$, such that

$$
f=P q+\sum_{j=1}^{p} r_{j} x_{n}^{p-j} .
$$

Proof. Set $x^{\prime}=\left(x_{1}, \ldots, x_{n-1}\right)$. We consider $f\left(x^{\prime}, x_{n}\right)$ and $f\left(x^{\prime}, \lambda_{1}\right)$ as elements of $C_{M}\left(\mathbb{R}^{n+p}, 0\right)$. Since $C_{M}\left(\mathbb{R}^{n+p}, 0\right)$ is closed under division by coordinates,

$$
f\left(x^{\prime}, x_{n}\right)-f\left(x^{\prime}, \lambda_{1}\right)=\left(x_{n}-\lambda_{1}\right) f_{1}(x, \lambda)
$$

with $f_{1} \in C_{M}\left(\mathbb{R}^{n+p}, 0\right)$ again. By repeating this process, we get 


$$
f(x)=g(x, \lambda)\left(x_{n}-\lambda_{1}\right) \ldots\left(x_{n}-\lambda_{p}\right)+\sum_{i=1}^{p} g_{i}\left(x^{\prime}, \lambda\right) x_{n}^{p-i},
$$

with $g \in C_{M}\left(\mathbb{R}^{n+p}, 0\right)$ and $g_{i} \in C_{M}\left(\mathbb{R}^{n-1+p}, 0\right), 1 \leq i \leq p$. We see that $g$ and $g_{i}, 1 \leq i \leq p$, are symmetric with respect to $\lambda_{1}, \ldots, \lambda_{p}$. By Corollary 3.4 , there exist $q \in C_{M}\left(\mathbb{R}^{n+p}, 0\right)$ and $r_{i} \in C_{M}\left(\mathbb{R}^{n-1+p}, 0\right), 1 \leq i \leq p$, such that

$$
g=\varphi^{*}(q), \quad g_{i}=\varphi^{*}\left(r_{i}\right)
$$

Then

$$
f=\varphi^{*}(f)=\varphi^{*}(q) \varphi^{*}(P)+\sum_{i} \varphi^{*}\left(r_{i}\right) x_{n}^{p-i}
$$

and this implies that

$$
f(x)=q(x, \lambda) P\left(x_{n}, \lambda\right)+\sum_{i} r_{i}\left(x^{\prime}, \lambda\right) x_{n}^{p-i}
$$

since $\varphi^{*}$ is injective. The uniqueness follows from the quasianalyticity of the class.

\section{References}

[BM] E. Bierstone and P. D. Milman, Resolution of singularities in Denjoy-Carleman classes, preprint.

[Ch] C. L. Childress, Weierstrass division in quasianalytic local rings, Canad. J. Math. 28 (1976), 938-953.

[EH] P. M. Eakin and G. A. Harris, When $\psi(f)$ convergent implies $f$ is convergent, Math. Ann. 229 (1977), 201-210.

[El] A. Elkhadiri, The theorem of the complement for a quasi subanalytic set, Studia Math. 161 (2004), 225-247.

[Ga] A. M. Gabrielov, Formal relations among analytic functions, Izv. Akad. Nauk SSSR Ser. Mat. 37 (1973), 1056-1090 (in Russian).

[Gl] G. Glaeser, Sur le théorème de préparation différentiable, in: Proc. Liverpool Singularities-Symposium, I (1969/70), Lecture Notes in Math. 192, Springer, Berlin, 1971, 121-132.

[Ko] H. Komatsu, The implicit function theorem of ultradifferentiable mappings, Proc. Japan Acad. Ser. A Math. Sci. 55 (1979), 69-72.

[RSW] J.-P. Rolin, P. Speissegger and A. J. Wilkie, Quasianalytic Denjoy-Carleman classes and o-minimality, J. Amer. Math. Soc. 16 (2003), 751-777.

Dipartimento di Matematica

Via Filippo Buonarroti 2

56127 Pisa, Italy

E-mail: broglia@dm.unipi.it pieroni@mail.dm.unipi.it
Département de Mathématiques et Informatique Faculté des Sciences Université Ibn Tofail

B.P. 133

Kenitra, Morocco E-mail: kabdelhafed@hotmail.com 